\title{
Metodología para evaluación de riesgo por flujo de detritos detonados por lluvia: caso Útica, Cundinamarca, Colombia
}

\author{
Methodology for debris flow risk assessment triggered by rainfall: Utica case study, \\ Cundinamarca, Colombia
}

Fecha de entrega: 21 de junio 2016

Fecha de aceptación: 2 de noviembre 2016

\section{Amanda Sepúlveda B., Jaime Patiño Franco y Carlos E. Rodríguez Pineda}

\begin{abstract}
Departamento de Ingeniería Civil, Pontificia Universidad Javeriana, Carrera 7 N 40-62, Bogotá D.C., Colombia, amanda.sepulveda@javeriana.edu.co,jaime.patino@javeriana.edu.co,crodriguezp@javeriana.edu.co
\end{abstract}

Los flujos de detritos son uno de los procesos de remoción en masa más destructivos en todo el mundo, dado que se generan en las zonas montañosas y se depositan en abanicos aluviales o llanuras aluviales ocupadas por asentamientos humanos, como ocurre en el caso de aplicación de esta investigación: Útica, en Cundinamarca, Colombia. En el presente trabajo se realiza una metodología para la evaluación de riesgo por flujo de detritos detonados por lluvia. En primera instancia se emplean sistemas de información geográfica para evaluar las zonas susceptibles a generar flujo de detritos, mediante el método de relación de frecuencias y considerando como factores detonantes la pendiente, litología, cobertura, curvatura y espesor del estrato superficial. La amenaza en zona de la cuenca se evalúa a través de umbrales de lluvia detonante y el mapa de susceptibilidad. Para la simulación del flujo de detritos se emplea el modelo matemático FLO-2D, determinando para cada periodo de retorno, el área de inundación y las profundidades y velocidades máximas del flujo sobre las potenciales zonas de depositación. Con los resultados de la simulación se realiza el mapa de amenaza en función de la intensidad del evento y la frecuencia de ocurrencia. La vulnerabilidad se determinó a partir de los indices de exposición y de resistencia, que son un indicativo del grado de exposición de las edificaciones y su comportamiento ante el evento amenazante. El riesgo de las edificaciones se plantea como una función de la amenaza, vulnerabilidad y el costo asociado al elemento expuesto.

Palabras clave: flujo de detritos, susceptibilidad, amenaza, vulnerabilidad fisica, indice de exposición, indice de resistencia, lluvia máxima, modelación matemática, caudal líquido, volumen de sedimentos, riesgo
Debris flows are one of the landslide mass processes more destructive worldwide, as they are generated in mountainous areas and deposited in alluvial fans or floodplains occupied by human settlements, as the application case of this research: Utica, Cundinamarca, Colombia. In this paper a methodology for risk assessment by debris flow triggered by rain is carried out. In the first instance, geographical information systems are used to assess susceptible areas to generate debris flow by the method of frequency ratio and considering triggering factors such as slope, lithology, coverage, curvature and thickness of the surface layer. Hazard basin area is evaluated through thresholds of trigger rain and susceptibility map. For the simulation of debris flow the mathematical model FLO-2D was used, determining for each return period flood area and maximum depths and flow velocities on potential areas of deposition. With simulation results the hazard map based on the intensity of the event and the occurrence frequency is performed. The vulnerability was determined from the indices of exposure and resistance, which are indicative of the degree of exposure of buildings and threatening behaviour before the event. Risk of buildings is presented as a function of hazard, vulnerability and the associated cost to the exposed element.

Keywords: debris flow, susceptibility, hazard assessment, physical vulnerability, exposure index, index of resistance, rain peak, mathematical modelling, liquid flow rate, volume of sediment, risk

\section{Introducción}

La región andina es un territorio susceptible a la ocurrencia de deslizamientos y flujos de detritos. En zonas húmedas predominan los deslizamientos, mientras que en zonas secas con menores precipitaciones y suelos desnudos o con escasa vegetación, predominan los flujos de detritos. 
La susceptibilidad a los flujos de detritos prima en los valles interandinos de Colombia, Perú, Ecuador y Bolivia, inclusive en zonas con muy pocas lluvias, pero con suelos desnudos o con poca vegetación como las vertientes del altiplano en Bolivia y del Pacífico de los Andes en Perú. Aunque en los cuatro países hay registros de impactos asociados con deslizamientos y flujos de detritos, la pérdida de vidas y viviendas por los segundos es comparativamente mayor en Colombia y Perú (Comunidad Andina, 2009).

La localización geográfica de Colombia en la región circumpacífica, en el punto de convergencia de tres placas tectónicas, a la vez que dentro de la zona intertropical, y el asentamiento de su población en la zona andina, la más inestable; hacen de este país Suramericano un escenario ideal para el estudio de la erosión, los procesos de remoción en masa, los terremotos y otros desastres de origen natural (Comunidad Andina, 2009).

De acuerdo con el estudio de amenaza realizado por Montero (1997), en el que recopila los movimientos en masa más importantes de Colombia desde la prehistoria, incluyendo los catastróficos, se concluye que el $40 \%$ de dichos eventos han involucrado flujos de detritos. El $30 \%$ de los flujos de detritos estudiados fueron catastróficos con grandes pérdidas de vidas humanas y pérdidas económicas cuantiosas.

Según los estudios realizados por Castellanos y González (1996), las causas de los eventos desastrosos (inundaciones, deslizamientos, avalanchas), son en un 96\% meteorológicas. El 56\% de estas causas se presentan por lluvias prolongadas o periodos lluviosos, el $37 \%$ por lluvias intensas de corta duración y en un menor porcentaje los huracanes.

En el reporte de emergencias obtenido el 16 de febrero de 2014 (SNGRD, 2014), en Colombia entre el 6 de mayo de 1998 al 7 de octubre de 2011 se han registrado 230 muertos, 195 heridos y 73 desaparecidos producidos por flujos de detritos y lodos ocurridos en diferentes regiones del país. Los antecedentes históricos de daños y muertes por flujos de detritos ocurridos en el país, sumado al gran crecimiento de la población, propiedades y actividades económicas ubicadas en el abanico aluvial de las quebradas, hacen necesario determinar el riesgo asociado a estos flujos e implementar medidas de mitigación que lo disminuyan.

\section{Caso de estudio}

Se ha tomado la cuenca de la Quebrada la Negra y el Municipio de Útica como caso de aplicación para la metodología propuesta, ya que en las laderas de dicha quebrada históricamente se han originado flujos de detritos de diferente intensidad, y la cabecera municipal ha sido afectada en diferentes ocasiones por dichos procesos, que han producido grandes pérdidas económicas y de vidas humanas. En la cuenca de la Quebrada La Negra las áreas de bosque han venido siendo reemplazadas aceleradamente por diferentes cultivos como caña, yuca y café, los cuales contribuyen a la alteración del material parental, constituido por rocas de origen sedimentario, de naturaleza arcillo arenosa, altamente deleznables y muy fracturadas, debido a que están localizadas en bloques o cuencas, limitadas entre sí por grandes fallas.

La alteración y degradación de la roca parental favorece el desprendimiento de los suelos superficiales de las laderas. Debido a las altas pendientes se facilita el transporte de materiales para alimentar la carga de sedimentos de los afluentes y de la Quebrada la Negra. Por su carácter torrencial, se tiene un alto potencial de arrastre de materiales, habiéndose originado en épocas de invierno flujos de detritos que se depositan en la población de Útica y causan daños en viviendas y afectación de la población.

La región para establecer el riesgo por flujo de detritos, abarca el abanico aluvial donde se localiza el casco urbano de la población de Útica en el departamento de Cundinamarca, está a $119 \mathrm{~km}$ de Bogotá D.C y al noroccidente de la misma, con una altitud de $497 \mathrm{msnm}$. Geográficamente Útica está delimitada en los $5^{\circ} 11^{\prime} 36,28^{\prime \prime}$ de latitud norte y $74^{\circ} 28^{\prime} 48,25^{\prime \prime}$ de longitud oeste, según el sistema de coordenadas WGS84.

La cuenca de la Quebrada La Negra está conformada por macizos rocosos de resistencia intermedia; Formaciones Útica, Socotá, Capotes, Hiló, Lidita Inferior, Nivel de Lutitas y Arenitas y por macizos rocosos de baja resistencia; Formación Trincheras y Grupo Guaguaquí (CAR, 2013).

\section{Metodología para la evaluación de la amenaza \\ Escalas}

El área mínima de una Unidad de Cálculo de Terreno - 
UCT, para realizar operaciones en SIG a escala adecuada, depende de la resolución espacial de los mapas. La UCT para efectos de cálculo es una unidad cuadrada, el tamaño de la unidad de cálculo de terreno está dada por la Tabla 1.

Tabla 1: Dimensiones de las unidades de cálculo del terreno

\begin{tabular}{|c|c|c|}
\hline \multirow{2}{*}{$\begin{array}{c}\text { Escala de } \\
\text { referencia }\end{array}$} & \multicolumn{2}{|c|}{$\begin{array}{c}\text { Dimensiones del pixel elemental - } \\
\text { UCT }\end{array}$} \\
\cline { 2 - 3 } & Longitud, $\mathrm{m}$ & Área, $\mathrm{m}^{2}$ \\
\hline $1: \mathrm{X}$ & $\mathrm{X} / 1000$ & $\mathrm{X}^{2} / 10^{6}$ \\
\hline $1: 250.000$ & 250 & 62500 \\
\hline $1: 100.000$ & 100 & 10000 \\
\hline $1: 25.000$ & 25 & 625 \\
\hline $1: 5.000$ & 5 & 25 \\
\hline
\end{tabular}

Para este estudio, el tamaño de la celda (pixel) es de $10 \mathrm{~m}$ que corresponde a $100 \mathrm{~m}^{2} \mathrm{y}$, las evaluaciones en el área urbana se realizarán con escala 1:5.000.

\section{Factores condicionantes}

Los factores condicionantes seleccionados para la evaluación de la susceptibilidad, corresponden a las siguientes variables:

- Pendiente y la naturaleza de los materiales geológicos (litología). Estas variables son los dos factores físicos que se consideran los componentes imprescindibles para evaluar la susceptibilidad de los movimientos de ladera (García y López, 2005). Han sido las más empleadas en la evaluación de susceptibilidad, habiendo sido incluidas de manera simultánea por Esper (2011, 2012, 2014), Moreiras (2004, 2009), Cardozo (2013), Gómez et al. (2013), Fídel y Zavala (2013), Mardones y Vidal (2001), entre muchos otros.

- Cobertura y uso del suelo. De acuerdo con Acosta (2006), esta variable incide significativamente en la aparición de flujos de detritos. Remondo (2001) y Haigh et al. (1988) comprobaron que esta variable es importante en el análisis de susceptibilidad.

- Curvatura. La curvatura es un factor determinante en la aparición de flujos de detritos porque refleja las áreas en las que los residuos tienden a acumularse (BlaisStevens y Behnia, 2016). La curvatura controla las zonas de acumulación o propagación de agua, lo que influye directamente en el grado de humedad y/o saturación del suelo y por ende, en la generación de flujos de detritos (Náquira, 2014).

\section{Análisis de susceptibilidad}

El análisis de susceptibilidad a la ocurrencia de flujo de detritos se obtuvo con la aplicación del método estadístico bivariado de relación de frecuencias. Esto corresponde a la razón aritmética entre el número de pixeles de clase (para cada variable) que es afectada por flujos de detritos y el área total de la clase, de la variable considerada. Los valores de la relación de frecuencia indican el nivel de correlación entre la ocurrencia de los flujos de detritos y las variables consideradas. De esta manera, para valores de relación de frecuencias $F_{\mathrm{r}}$ mayores que 1, la correlación entre la ocurrencia de flujos de detritos y las variables consideradas, es alta, mientras que valores de $F_{\mathrm{r}}$ menores que 1 indican una baja correlación (Esper, 2011).

La relación de frecuencias $F_{\mathrm{r}}$ se calcula con la expresión (1) de Bonham-Carter (1994):

$$
F_{r}=\frac{\frac{N_{p i x}(F D)}{N_{p i x}(F D t)}}{\frac{N_{p i x}(A)}{N_{p i x}(A t)}}
$$

donde $N_{\text {pix }}(F D)$ es el número de pixeles que contienen flujos de detritos en la clase de la variable temática, $N_{\text {pix }}(F D t)$ es el número de pixeles totales que contienen flujos de detritos, $N_{\text {pix }}(A)$ es el número total de pixeles para la clase de la variable considerada, $N_{\text {pix }}(A t)$ es el número total de pixeles dentro de la zona de estudio. El índice de susceptibilidad a la ocurrencia de flujos de detritos ISFD de cada celda, se obtiene mediante la expresión (2).

ISFD $=$ litología $_{\mathrm{r}}+$ curvatura $_{\mathrm{r}}+$ pendiente $_{\mathrm{r}}+$ cobertura $_{\mathrm{r}}+$ espesor del estrato $_{\mathrm{r}}$

Donde litología $a_{\mathrm{r}}$ es el coeficiente $F_{\mathrm{r}}$ de la variable litología; curvatura $_{\mathrm{r}}$ es el coeficiente $F_{\mathrm{r}}$ de la variable curvatura; pendiente $\mathrm{r}_{\mathrm{r}}$ es el coeficiente $F_{\mathrm{r}}$ de la variable pendiente y así sucesivamente.

Para el mapa susceptibilidad por flujo de detritos obtenido de la cuenca en estudio y mostrado en la Figura 1, los rangos fueron establecidos por el método de Cuantil, para las tres categorías de bajo, medio y alto, utilizando el Sistema de Información Geográfica Arcgis.

Elárea de estudio posee 168 flujos de detritos inventariados. 
Para el análisis de susceptibilidad se emplearon 120, el resto fueron separados de la evaluación para verificación espacial del mapa de susceptibilidad realizado. Para la validación de la predicción, los 48 flujos restantes se superpusieron en el mapa de susceptibilidad obtenido, quedando el $83.30 \%$ de los movimientos en zona de susceptibilidad alta, $14.53 \%$ en susceptibilidad media y $2.17 \%$ en susceptibilidad baja.

Esta clasificación de los flujos de detritos para validación, en los niveles de susceptibilidad definidos, indica el grado de predicción del método propuesto. De esta forma, cuando la mayor parte de los movimientos quedan clasificados en rangos de susceptibilidad bajos, indicará que el método no ha reproducido las condiciones de inestabilidad; de otra parte, si los flujos de detritos se localizan en rangos altos, esto denotará un mejor ajuste de la predicción. Dado que la mayoría de los movimientos empleados en la validación quedan clasificados en nivel alto de susceptibilidad, este es un indicador del buen ajuste de la predicción (83.30\%).

\section{La lluvia como evento detonante de flujo de detritos}

El desarrollo metodológico pretende la determinación de umbrales de lluvias críticas como factor detonante de flujos de detritos, e integrarlas con el mapa de susceptibilidad para así generar un modelo de pronóstico en tiempo real de amenazas por flujo de detritos. Se determinan inicialmente los umbrales de lluvia crítica detonante de flujo de detritos mediante los desarrollos teóricos propuestos por Mayorga (2003) y los resultados se contrastan con la propuesta de Kanji et al. (1997, 2001).

Mayorga (2003) propuso el método de Lluvia Crítica para la determinación de los umbrales de lluvias detonantes de deslizamientos, la cual se basa en el análisis de la precipitación acumulada o antecedente de días previos a la ocurrencia del evento, y está formulada en términos probabilísticos.

Por su parte, Kanji et al. (1997, 2001) establecieron umbrales de lluvia detonantes de flujos de detritos con un inventario de deslizamientos en diferentes regiones de Latinoamérica, donde predominan los suelos residuales, incluyendo procesos de flujos en Colombia.

Para evaluar la lluvia como evento detonante de deslizamientos empleando la metodología de Mayorga (2003) deben seguirse la secuencia que se relaciona a continuación:

\section{Análisis de la lluvia antecedente - larga duración}

Se deben identificar las estaciones pluviométricas en la zona de influencia de la cuenca en estudio y obtener los registros de precipitación diaria antecedente a uno o varios eventos históricos de flujo de detritos (hasta los 3 a 6 meses anteriores).

Realizar la gráfica de la lluvia acumulada para cada evento, donde el eje de abscisas corresponda a la duración en días y el eje de ordenadas corresponda a la precipitación acumulada en mm. Se toma como inicio el día en que ocurrió el evento de flujo de detritos; la curva debe realizarse de forma regresiva hasta obtener periodo seco, donde la precipitación sea cero, punto que determina la precipitación acumulada.

Se emplea el modelo de regresión logística (3) para establecer la relación entre la lluvia acumulada y la lluvia evento y definir el umbral de lluvia detonante.

$$
P i=\frac{1}{1+e^{-\left(\beta_{0}+\beta_{1} X_{1}+\cdots+\beta_{p} X_{p}\right)}}
$$

Posteriormente, los umbrales establecidos por Kanji et al.(1997, 2003) (Figura 2), se comparan con los periodos de retorno establecidos en las curvas Intensidad-DuraciónFrecuencia, para la cuenca la Quebrada La Negra, con el fin de determinar el periodo correspondiente al detonante de precipitación.

\section{Frecuencia de ocurrencia de lluvias}

Kanji et al. (1997, 2001) encontraron que importantes deslizamientos ocurren con altos valores de precipitación en largos períodos de tiempo, que van de días a semanas y los flujos de detritos ocurren con gran intensidad de precipitación en pocos períodos de tiempo, después de un período precedente de lluvias que podría ser de baja intensidad. Los umbrales establecidos por Kanji et al. (2003) y mostrados en la Figura 2, se compararon con los periodos de retorno establecidos en las curvas IntensidadDuración-Frecuencia, para la cuenca la Quebrada La Negra, con el fin de determinar el periodo correspondiente al detonante de precipitación. 
Al estudiar la Figura 2 se establecen los umbrales de precipitación como detonante de flujo de detritos, para el caso de la cuenca Quebrada La Negra en Útica (Cundinamarca). Los umbrales de lluvia detonante establecidos para la cuenca la Quebrada La Negra con base en las curvas de Kanji, se presentan en la Tabla 2.

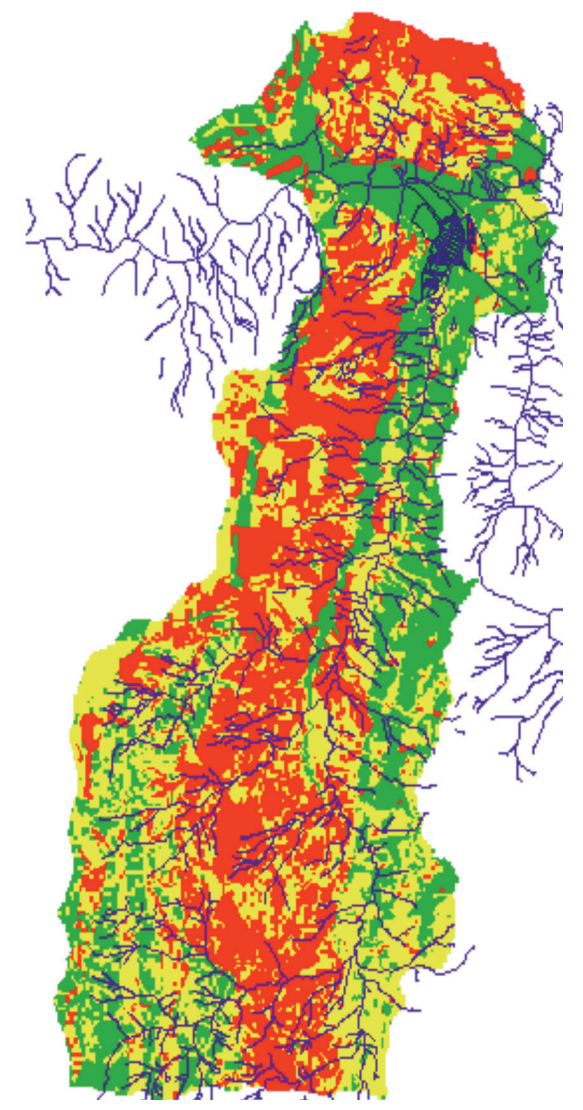

Figura 1: Mapa de la susceptibilidad de flujo de detritos en la cuenca Quebrada La Negra en Útica, Cundinamarca

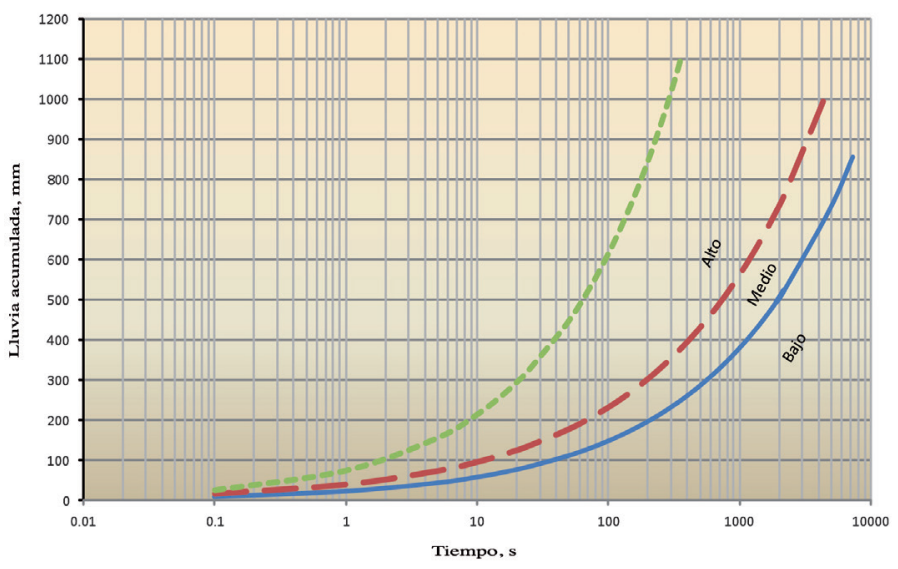

Figura 2: Lluvia acumulada versus el tiempo detonante del flujo de detritos y grandes deslizamientos (modificado de Kanji et al., 1997, 2003). La línea inferior TL representa un umbral para remociones en masa
Tabla 2: Umbrales de precipitación como detonante de flujo de detritos en la Quebrada La Negra

\begin{tabular}{|c|c|}
\hline $\begin{array}{c}\text { Umbral de precipitación acumulada, } \\
\mathrm{mm}\end{array}$ & Clase \\
\hline$<470$ & Bajo \\
\hline $470-700$ & Medio \\
\hline$>700$ & Alto \\
\hline
\end{tabular}

Con el mapa de susceptibilidad de la cuenca y los umbrales de precipitación se obtiene el mapa de amenaza de la cuenca mostrado en la Figura 3.

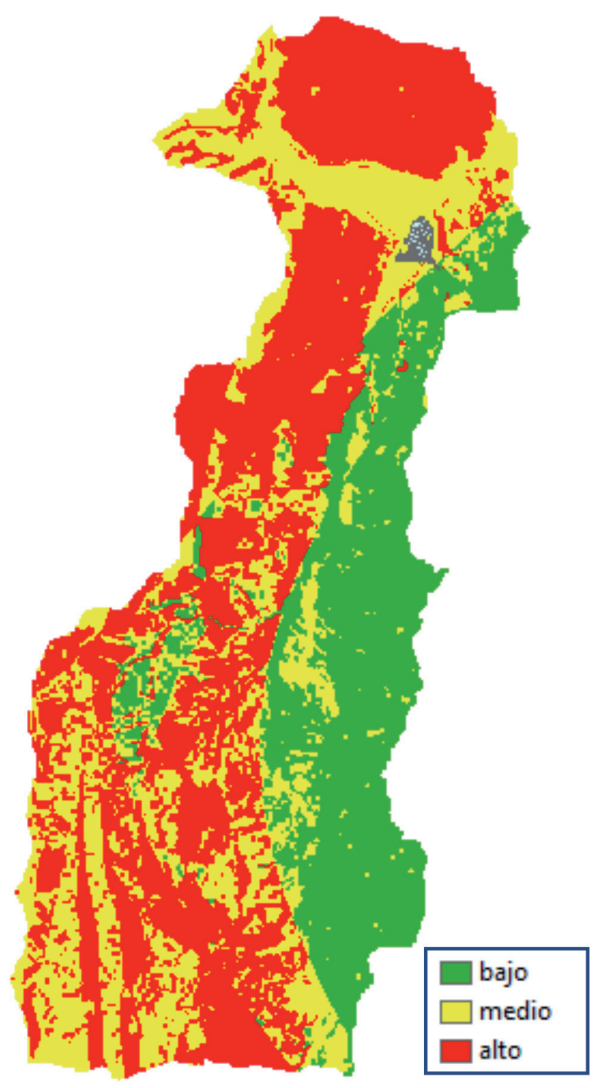

Figura 3: Mapa de amenaza de la cuenca Quebrada La Negra en Útica, Cundinamarca

\section{Modelación matemática del flujo de detritos}

Los flujos de detritos son movimientos complejos que no pueden representarse por medio de los métodos que se aplican comúnmente para fluidos como el agua. Esto se debe a que la alta concentración volumétrica hace que estos fluidos se comporten como fluidos no-Newtonianos, en los que es necesario que actúe un esfuerzo superior a un determinado esfuerzo crítico, para que el fluido se ponga 
en movimiento (Valderrama, 2006). Según O’Brien et al. (2006), el FLO-2D es un modelo de diferencias finitas en dos dimensiones que simula inundación de agua limpia, flujos de lodo y flujos de detritos en los abanicos aluviales y llanuras de inundación urbanas. El programa transita el volumen del flujo a través de una serie de celdas sobre la llanura de inundación o a través de segmentos para flujo canalizado. La propagación del flujo es controlado por la topografía y la resistencia al flujo. El tránsito en dos dimensiones se logra a través de integración numérica de las ecuaciones de movimiento y conservación del volumen del fluido, bien sea para inundación o para flujo de detritos.

\section{Ecuaciones de gobierno}

Las ecuaciones generales constitutivas son la ecuación de continuidad y las ecuaciones de movimiento (ecuación de momentum de onda dinámica). En general, la extensión de los flujos de detritos predomina más que su altura en la escala, y la translación predomina sobre la rotación. Es razonable asumir que las ecuaciones de gobierno pueden ser integradas a lo largo de la profundidad. En un sistema de coordenadas fijo $(x, y, z)$ con $z$ en sentido opuesto a la dirección de la gravedad, las ecuaciones de gobierno pueden ser reducidas a las relaciones con la profundidad promedio en el plano $x-y$.

La expresión (4) corresponde a la ecuación de continuidad.

$$
\frac{\partial h}{\partial t}+\frac{\partial h}{\partial x} V_{x}+\frac{\partial h}{\partial y} V_{y}=i
$$

Las ecuaciones bidimensionales de movimiento están dadas por:

$$
\begin{aligned}
& S_{f x}=S_{o x}-\frac{\partial h}{\partial x}-\frac{V_{x}}{g} \frac{\partial V_{x}}{\partial x}-\frac{V_{y}}{g} \frac{\partial V_{x}}{\partial y}-\frac{1}{g} \frac{\partial V_{x}}{\partial t} \\
& S_{f y}=S_{o y}-\frac{\partial h}{\partial y}-\frac{V_{y}}{g} \frac{\partial V_{y}}{\partial y}-\frac{V_{x}}{g} \frac{\partial V_{y}}{\partial x}-\frac{1}{g} \frac{\partial V_{y}}{\partial t}
\end{aligned}
$$

Donde $h$ es la profundidad del flujo.

$V_{\mathrm{x}}$ y $V_{\mathrm{y}}$ son las componentes de la velocidad a profundidad media a lo largo de los ejes coordenados $x$ e $y$. Los excesos de intensidad de lluvia $i$ pueden ser diferentes de cero sobre la superficie del flujo, los componentes de la pendiente de fricción $S_{\mathrm{fx}}$ y $S_{\mathrm{fy}}$ se consideran como una función de la pendiente del canal $S_{\text {ox }}$ y $S_{\text {oy }}$, el gradiente de presiones y la aceleración convectiva y local. Una aproximación de la onda difusiva para las ecuaciones de movimiento se define despreciando la última de las tres componentes de aceleración. De igual manera, a partir de la ecuación de momentum, se obtiene una representación de la onda cinemática eliminando el término gradiente de presión. La opción de seleccionar la onda difusiva o la onda dinámica está disponible en el programa FLO-2D (O'Brien et al., 1993).

\section{Módelo reológico}

El comportamiento reológico de los flujos de sedimentos hiperconcentrados envuelve la interacción de varios procesos físicos complejos. El comportamiento no Newtoniano es controlado en parte por la cohesión entre los sedimentos finos de partículas. Esta cohesión contribuye al esfuerzo de cedencia $\tau_{y}$, que debe ser sobrepasado por un esfuerzo aplicado a fin de iniciar el movimiento del fluido (O’Brien et al., 1993).

Otros procesos físicos que se consideraron son: los esfuerzos de corte viscosos, el esfuerzo de corte debido a la turbulencia y los esfuerzos dispersivos debido a la colisión de los clastos. El esfuerzo de corte total en flujos de sedimentos hiperconcentrados, incluyendo flujos de detritos se muestra en (7), se calcula como la suma de cinco componentes del esfuerzo de corte:

$\tau=\tau_{c}+\tau_{m c}+\tau_{v}+\tau_{t}+\tau_{d}$

donde $\tau_{c}$ es el esfuerzo de fluencia, $\tau_{\mathrm{mc}}$ es el esfuerzo de corte Mohr - Coulomb, $\tau_{v}$ es el esfuerzo cortante viscoso, $\tau_{t}$ es el esfuerzo cortante turbulento y $\tau_{d}$ es el esfuerzo cortante dispersivo. La expresión (7) puede ser expresada en términos de tasas de deformación $\mathrm{d} v / \mathrm{d} y$ como se observa en (8), con el modelo reológico cuadrático.

$\tau=\tau_{y}+\eta\left(\frac{d v}{d y}\right)+C\left(\frac{d v}{d y}\right)^{2}$

Donde $\tau_{\mathrm{y}}=\tau_{\mathrm{c}}+\tau_{\mathrm{mc}}, d v / d y$ es el gradiente de velocidad, $\eta$ es la viscosidad dinámica, $C$ es el coeficiente del esfuerzo cortante inercial e $\tau_{y}$ es el esfuerzo de cadencia. Los 
principales datos de entrada del modelo son los siguientes:

- Topografía digital del terreno, por medio del modelo digital de elevación

- Geometría cauce natural

- Valores de la rugosidad del cauce y de la planicie de inundación

Hidrogramas de entrada (líquidos y sólidos).

Los caudales líquidos obtenidos de modelos hidrológicos tales como HEC-HMS y caudales sólidos en función de la amenaza alta de ocurrencia de flujo de detritos en la cuenca de estudio.

Propiedades reológicas de la mezcla agua-sedimento.

Para la calibración del modelo FLO-2D, se requiere comparar el volumen de detritos o las alturas de depositación reportadas por el modelo con el volumen de detritos o los tirantes del flujo medidos para eventos históricos. En este caso, se obtuvieron los tirantes de altura alcanzados por el flujo de detritos en el casco urbano de Útica, en el evento ocurrido en el año 1988, a través del Servicio Geológico Nacional.

Los resultados de la calibración del modelo mostraron que hay una influencia directa del coeficiente de rugosidad de Manning sobre la profundidad y la velocidad del flujo de detritos. Al aumentar el coeficiente de Manning la altura del flujo se incrementa, pero la velocidad disminuye. En cuanto a la concentración volumétrica $C_{\mathrm{v}}$, existe un punto de inflexión, para el cual a mayores valores de $C_{\mathrm{v}}$, la altura de depositación se incrementa, pero para valores menores la altura del flujo permanece estable.

\section{Amenaza en el casco urbano}

El modelo FLO-2D evalúa el nivel de amenaza para flujo de detritos de acuerdo con la metodología propuesta por García et al. (2002) y García y López (2005); según la cual, la amenaza por flujo de detritos está determinada por la probabilidad de ocurrencia del evento y su intensidad, siendo esta última función del tirante de profundidad y la velocidad del flujo, como se muestra en la Tabla 3 y la Figura 4.

Con los datos obtenidos en las modelaciones para los diferentes períodos de retorno, en el programa FLO-2D, se ejecuta la subrutina hazard y se obtiene el mapa de amenaza por flujo de detritos que se muestra en la Figura 5.

Tabla 3: Definición de la intensidad de flujo de detritos (García et al., 2002; García y López, 2005)

\begin{tabular}{|c|c|c|}
\hline $\begin{array}{c}\text { Intensidad de } \\
\text { flujo }\end{array}$ & $\begin{array}{c}\text { Máxima profundi- } \\
\operatorname{dad} h, \mathrm{~m}\end{array}$ & $\begin{array}{c}\text { Velocidad máxima } V \\
\text { por } h, \mathrm{~m}^{2} / \mathrm{s}\end{array}$ \\
\hline Alta & $h>1.0$ & $V h>1.0$ \\
\hline Media & $0.2<h<1.0$ & $0.2<V h<1.0$ \\
\hline Baja & $0.2<h<1.0$ & $V h<0.2$ \\
\hline
\end{tabular}
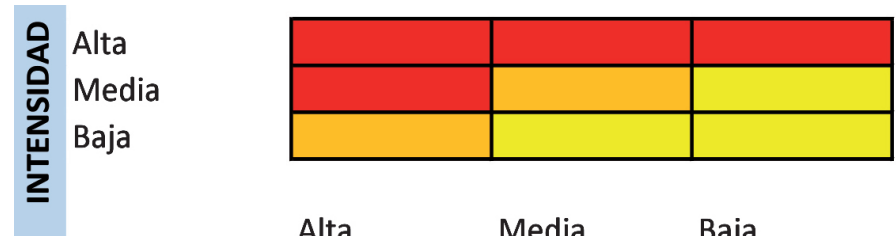

Alta Media Baja

PROBABILIDAD

\begin{tabular}{cccc}
$\begin{array}{c}\text { Periodo de } \\
\text { retorno }\end{array}$ & 10 & 25 & 50 \\
\hline $\begin{array}{c}\text { Probabilidad } \\
\text { de excedencia }\end{array}$ & $10.00 \%$ & $4.00 \%$ & $2.00 \%$
\end{tabular}

Figura 4: Clasificación de la amenaza en función de la intensidad del evento y la probabilidad de ocurrencia, adaptado de García et al. (2002)

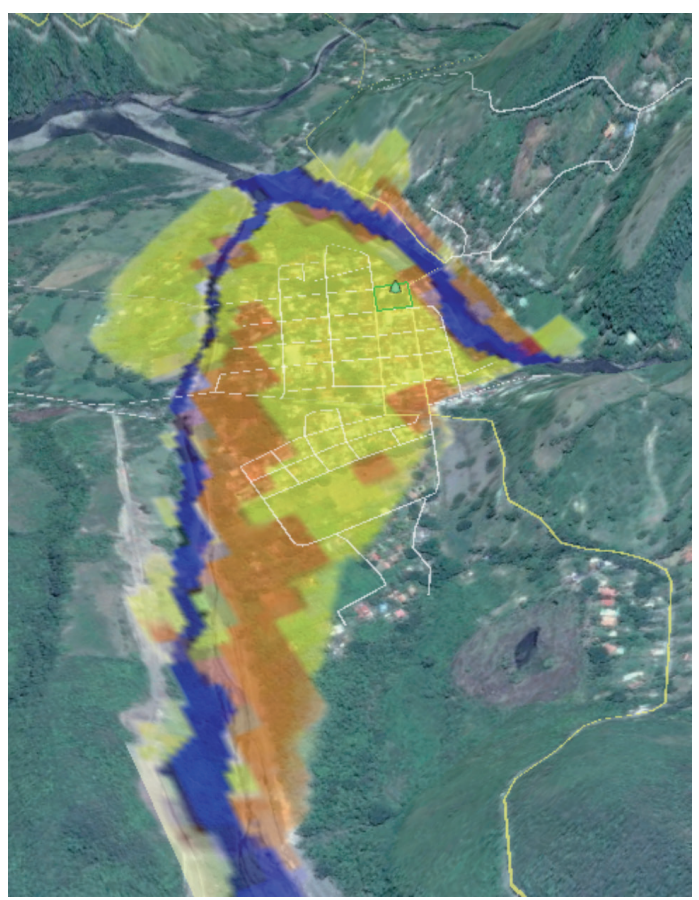

Figura 5: Mapa de amenaza por flujo de detritos en Útica, Cundinamarca

El casco urbano de Útica presenta en mayor proporción 
dos zonificaciones de amenaza las cuales corresponden a media y baja; siendo la amenaza media la localizada en cercanías de las márgenes de la quebrada La Negra y río Negro, y la baja en la parte central del casco urbano. La amenaza alta se localiza en los terrenos aledaños a las márgenes de la quebrada La Negra y río Negro.

\section{Metodología para evaluación de la vulnerabilidad}

Para la metodología propuesta, el índice de vulnerabilidad física mostrado en (9) corresponde a la expresión matemática propuesta por González y Millán (1998) y modificada por Lara (2002), para expresar la vulnerabilidad en términos de exposición y resistencia.

$$
I V F=\left(1-I R^{I E}\right)^{(1-I E)}
$$

Donde $I V F$ es el índice de vulnerabilidad física, $I R$ es el índice de resistencia e $I E$ es el índice de exposición. Cuando $I E=0, I V F=0$.

\section{Índice de resistencia}

El índice de resistencia se refiere a la capacidad de una edificación para resistir el evento amenazante. Para efectos de desarrollo de la metodología, este índice se ha expresado en términos del número de pisos de la edificación, su sistema estructural y las características de las aberturas en fachada.

\section{Índice de exposición}

El índice de exposición considera la vulnerabilidad que se puede presentar en un elemento expuesto, una vez materializada la amenaza, en virtud de su proximidad al evento amenazante y la intensidad del mismo. Este índice se expresa en términos de los parámetros: altura de depositación del flujo y presión de impacto, obtenidas de la modelación del flujo en el programa FLO-2D. El índice de exposición se adapta de las formulaciones propuestas por Quan Luna et al. (2011), empleando los valores críticos para presión de impacto propuestos por Keiler et al. (2006) para construcciones ligeras de mamposteria, y una altura crítica de $1.0 \mathrm{~m}$. Considerando que para el caso del flujo de detritos, se ha observado un incremento súbito del grado de pérdidas una vez que la altura de depositación del flujo supera este valor, lo cual considera la importancia de las características de las aberturas de la edificación, especialmente las ventanas, que están relacionadas con esta altura crítica (Papathoma et al., 2012). La expresión final para el índice de exposición corresponde a la siguiente expresión (10):

Para $P i \leq 6.45 \mathrm{kPa}$ y $h_{\mathrm{df}}<1 \mathrm{~m}$

$I E=\frac{1.49\left(h_{d f} / 0.693\right)^{1.938}}{1+\left(h_{d f} / 0.693\right)^{1.938}} \frac{1.596(P / 4.845)^{1.808}}{1+(P / 4.845)^{1.808}}$

Para $P i>6.45 \mathrm{kPa}$ y $h_{\mathrm{df}}>1 \mathrm{~m}$

$I E\left(h_{\mathrm{df}}, P\right)=1$

\section{Índice de vulnerabilidad física}

Con los índices de resistencia y exposición, se obtiene el índice de vulnerabilidad física IVF para cada edificación, aplicando (9). Los diferentes tipos de daños o efectos sobre las edificaciones que se esperarían como resultado de la ocurrencia del flujo de detritos, para los diferentes periodos de retorno, son los relacionados en la Tabla 4.

Tabla 4: Clasificación vulnerabilidad física y daños esperados, adaptado de Chaparro (2005) y Leone (1996).

\begin{tabular}{|c|c|c|c|c|}
\hline $\begin{array}{l}\text { Índice de } \\
\text { Vulnerabi- } \\
\text { lidad Física }\end{array}$ & $\begin{array}{l}\text { Categoría } \\
\text { de Vulne- } \\
\text { rabilidad }\end{array}$ & Color & $\begin{array}{l}\text { Rangos } \\
\text { de daño }\end{array}$ & Tipo de daño \\
\hline $0-0.10$ & Baja & & $0.01-0.1$ & $\begin{array}{l}\text { Daños no estructu- } \\
\text { rales leves. No se } \\
\text { afecta la estabilidad. } \\
\text { Mobiliario dañado }\end{array}$ \\
\hline $0.10-0.4$ & Media & & $0.1-0.4$ & $\begin{array}{l}\text { Fisuras en muros, } \\
\text { estabilidad no afec- } \\
\text { tada, reparaciones } \\
\text { no urgentes }\end{array}$ \\
\hline \multirow{3}{*}{$0.4-1.0$} & \multirow{3}{*}{ Alta } & & $0.4-0.6$ & $\begin{array}{l}\text { Deformaciones se- } \\
\text { veras, grietas muy } \\
\text { abiertas, grietas en } \\
\text { los elementos es- } \\
\text { tructurales. Esta- } \\
\text { bilidad afectada. } \\
\text { Puertas y ventanas } \\
\text { inutilizables. Eva- } \\
\text { cuación necesaria. }\end{array}$ \\
\hline & & & $0.6-0.8$ & $\begin{array}{l}\text { Fracturas estructu- } \\
\text { rales, destrucción } \\
\text { parcial, es necesario } \\
\text { evacuar }\end{array}$ \\
\hline & & & $0.8-1.0$ & $\begin{array}{l}\text { Destrucción parcial } \\
\text { o total, evacuación } \\
\text { necesaria, recons- } \\
\text { trucción total. }\end{array}$ \\
\hline
\end{tabular}


Los índices de vulnerabilidad para la población de Útica, para los periodos de retorno de 10, 25 y 50 años fueron mapeados en Arcgis y se muestran en las Figuras 6, 7 y 8.

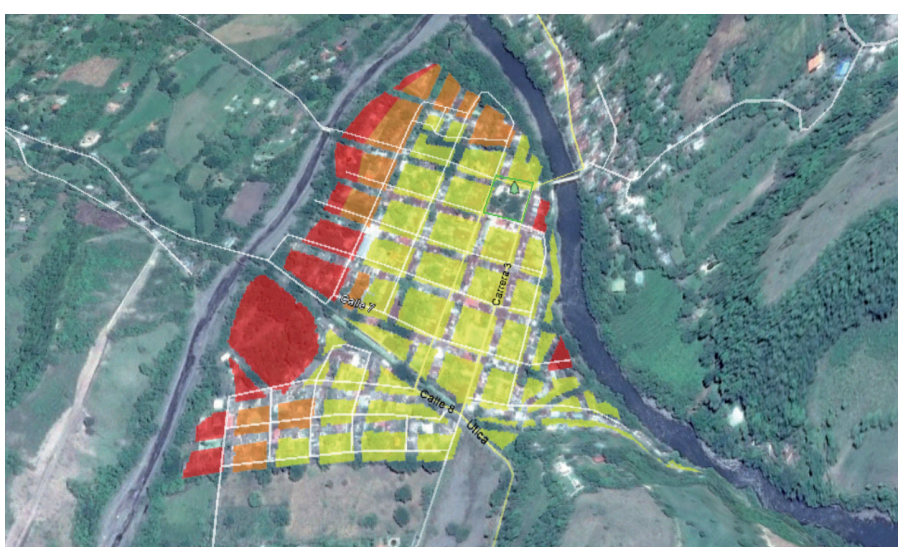

Figura 6: Vulnerabilidad física casco urbano Útica para un periodo de retorno de 10 años

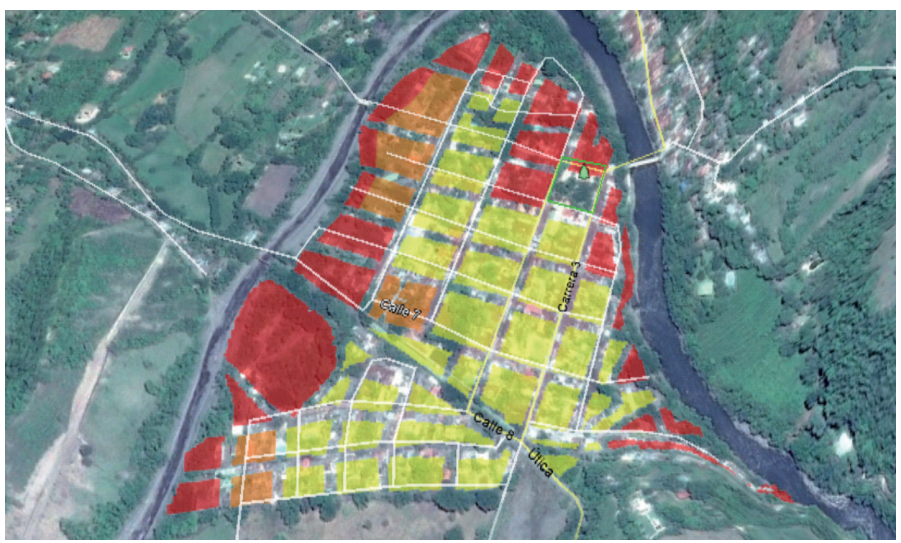

Figura 7: Vulnerabilidad física casco urbano Útica para un período de retorno de 25 años

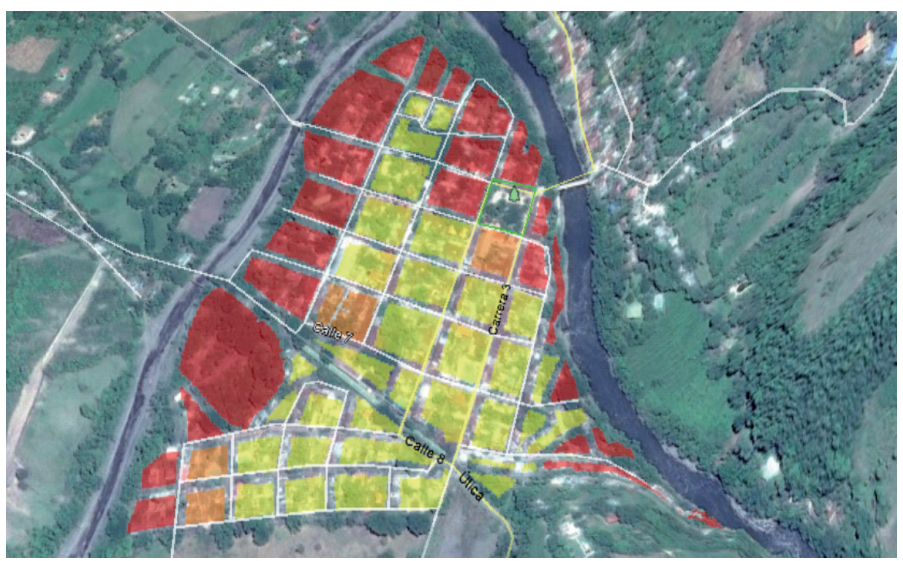

Figura 8: Vulnerabilidad física casco urbano Útica para un periodo de retorno de 50 años
En la Tabla 5, se observan las afectaciones que se pueden presentar en las edificaciones para cada período de retorno.

Tabla 5: Vulnerabilidad de las edificaciones en el casco urbano de Útica

\begin{tabular}{|c|c|c|c|}
\hline $\begin{array}{c}\text { Período de } \\
\text { retorno, } \\
\text { años }\end{array}$ & $\begin{array}{c}\text { Deformaciones } \\
\text { severas, hasta } \\
\text { destrucción par- } \\
\text { cial o total, } \%\end{array}$ & $\begin{array}{c}\text { Fisuras leves } \\
\text { sin afectación } \\
\text { de la estabili- } \\
\text { dad, } \%\end{array}$ & $\begin{array}{c}\text { Daños no } \\
\text { estructurales } \\
\text { leves, \% }\end{array}$ \\
\hline 10 & 13 & 8 & 79 \\
\hline 25 & 23 & 7 & 70 \\
\hline 50 & 36 & 7 & 57 \\
\hline
\end{tabular}

\section{Metodología para la evaluación del riesgo}

El riesgo se evalúa como:

Riesgo $=H I V \$$

donde $H$ es la amenaza, $I V$ es un índice de vulnerabilidad y $\$$ es el costo asociado a la edificación. El costo asociado a la edificación corresponde al valor actual del inmueble $V A$, este valor se calcula con el método de Ross - Heidecke, que considera la depreciación de la edificación en función de su estado de conservación y de mantenimiento. El método se basa en las consideraciones de que la depreciación es la pérdida de valor que no puede ser recuperada con gastos de mantenimiento y que las reparaciones pueden aumentar la durabilidad de la edificación.

Con base en lo establecido por la Australian Geomechanics Society AGS (australiangeomechanics. org), la cuantificación de pérdidas de las estructuras como edificaciones, se obtienen con el costo aproximado de los daños sufridos, que se define como el porcentaje de daño multiplicado por el valor comercial del predio, incluyendo el terreno y la edificación con su estado de conservación antes del evento. En función de la amenaza y la vulnerabilidad se define el daño esperado, cuando dicho producto presenta valores mayores entre 0.8 y 1.0 , se estima que la edificación requiere demolerse y volverse a reconstruir por lo que el porcentaje indicativo del daño es del 200\%. En la Tabla 6, se presenta el criterio la valoración del costo del daño en porcentaje. 
Tabla 6: Porcentaje indicativo del daño

\begin{tabular}{|c|c|c|c|c|}
\hline \multirow{2}{*}{\multicolumn{2}{|c|}{\begin{tabular}{|l|} 
Amenaza \\
x Vulne- \\
rabilidad
\end{tabular}}} & $\begin{array}{l}\text { Costo apro- } \\
\text { ximado }\end{array}$ & \multirow[b]{2}{*}{ Descripción } & \multirow[b]{2}{*}{ Descriptor } \\
\hline & & $\begin{array}{l}\text { Porcentaje } \\
\text { indicativo } \\
\text { del daño, }\end{array}$ & & \\
\hline 0.8 & 1 & 200 & $\begin{array}{l}\text { Estructura completamen- } \\
\text { te destruida y/o daños a } \\
\text { gran escala que requieren } \\
\text { reconstrucción. }\end{array}$ & $\begin{array}{l}\text { Catastró- } \\
\text { fico }\end{array}$ \\
\hline 0.6 & 0.8 & 80 & Grandes daños en la ma- & Mayor \\
\hline 0.4 & 0.6 & 60 & $\begin{array}{l}\text { y/o se extiende más allá } \\
\text { de los límites de los sitios } \\
\text { que requieren trabajos de } \\
\text { reconstrucción significa- } \\
\text { tivos. }\end{array}$ & Mayor \\
\hline 0.1 & 0.4 & 40 & $\begin{array}{l}\text { Daño moderado a algunas } \\
\text { partes de la estructura, y/o } \\
\text { una parte significativa. }\end{array}$ & Medio \\
\hline 0.01 & 0.1 & 10 & $\begin{array}{l}\text { Daño limitado a una parte } \\
\text { de la estructura. }\end{array}$ & Menor \\
\hline
\end{tabular}

Una vez determinado el criterio de la cuantificación del daño, se realiza la categorización del riesgo con los niveles alto A, medio B y bajo B por el método de cuantiles. En la Tabla 7, se presenta la matriz de riesgo, donde se establecen las relaciones entre la categorización del nivel de riesgo y la propiedad.

Tabla 7: Matriz de riesgo

\begin{tabular}{|l|l|}
\hline \multicolumn{1}{|l|}{ Nivel del riesgo } & Descripción \\
\hline Alto & $\begin{array}{l}\text { Inaceptable sin tratamiento. Extensa } \\
\text { investigación detallada para la investigación, } \\
\text { planificación y ejecución del tratamiento. } \\
\text { Opciones esenciales para reducir el riesgo } \\
\text { a nivel bajo puede ser demasiado caro y no } \\
\text { es práctico. Los trabajos de reconstrucción } \\
\text { cuestan más que el valor de la propiedad. }\end{array}$ \\
\hline Medio & $\begin{array}{l}\text { Puede ser tolerada en ciertas circunstancias } \\
\text { (sujeto a la aprobación por los entes } \\
\text { reguladores) pero requiere investigación, } \\
\text { planificación e implementación de las } \\
\text { opciones de tratamiento para reducir el } \\
\text { riesgo a bajo. Las opciones de tratamiento } \\
\text { para reducir a un menor riesgo debe ser } \\
\text { implementadas tan pronto como sea posible. }\end{array}$ \\
\hline Bajo & $\begin{array}{l}\text { Por lo general, aceptables para los entes } \\
\text { reguladores. El tratamiento ha sido necesario } \\
\text { para reducir el riesgo a este nivel, se requiere } \\
\text { el mantenimiento continuo. }\end{array}$ \\
\hline
\end{tabular}

Para el casco urbano de la población de Útica en Cundinamarca, los mapas de riesgo económico por flujo de detritos para los períodos de retorno de 10, 25 y 50 años, se presentan en las Figuras 9, 10 y 11.

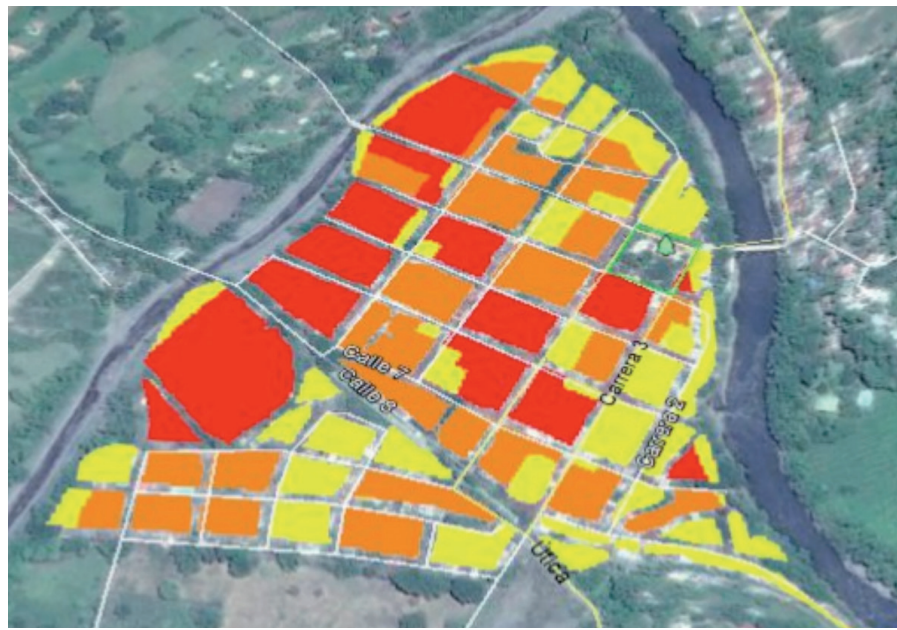

Figura 9: Riesgo casco urbano Útica para un periodo de retorno de 10 años

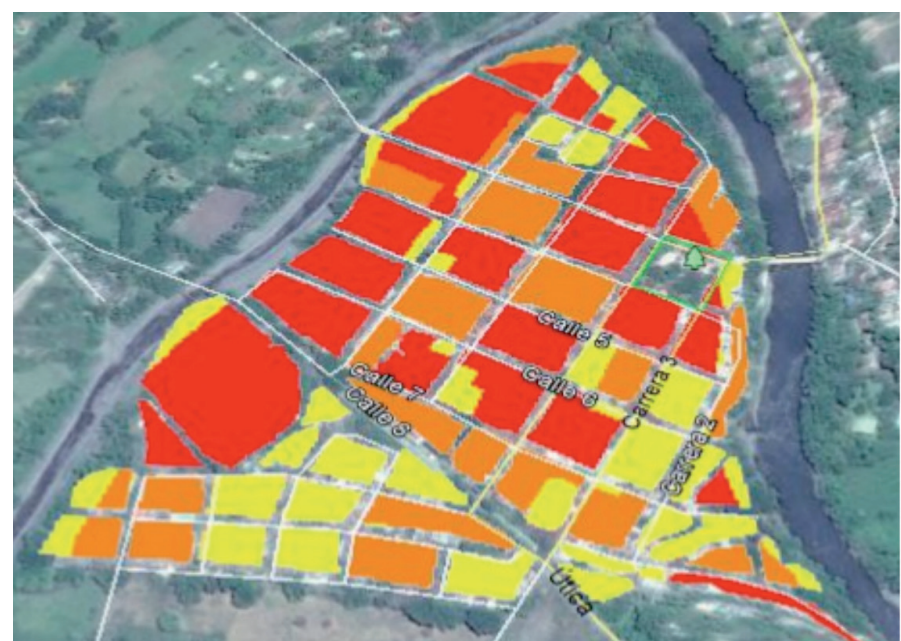

Figura 10: Riesgo casco urbano Útica para un periodo de retorno de 25 años

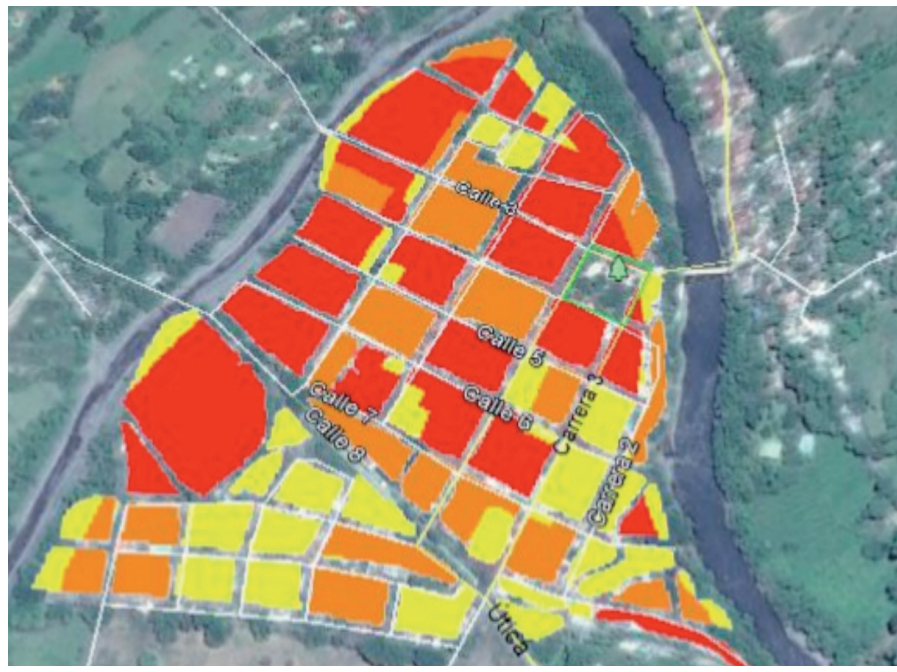

Figura 11: Riesgo casco urbano Útica para un periodo de retorno de 25 años 


\section{Discusión de resultados}

El análisis de susceptibilidad a la ocurrencia de flujo de detritos se realizó mediante la implementación del método estadístico bivariante que se fundamenta en el análisis superpuesto entre el mapa de inventario de flujo de detritos y los mapas de los factores condicionantes considerados. El modelo de susceptibilidad así considerado se generó a partir de las variables: pendiente, litología, curvatura, cobertura, espesor del estrato superficial y el inventario de flujos de detritos. Para todas las variables, se presentaron clases que tienen correlaciones importantes con la ocurrencia de flujos de detritos, aunque las clases donde se presentan mayores correlaciones son las de las variables cobertura y litología.

De acuerdo con la revisión bibliográfica adelantada, el espesor del estrato superficial no había sido considerado anteriormente en la elaboración de mapas de susceptibilidad, sin embargo los valores obtenidos indican que existe una correlación entre los rangos de valores que toma esta variable y la ocurrencia de flujos de detritos. Eso resulta acorde al planteamiento de ISSMFE (1997) que establece que el espesor del suelo superficial es uno de los factores que se deben tener en cuenta para la evaluación de susceptibilidad a deslizamientos detonados por lluvia.

Del mapa de pendientes se observa que las pendientes en el área de estudio varían entre 0 y $56^{\circ}$, siendo las pendientes mayores a $35^{\circ}$ las que muestran mayor asociación con el flujo de detritos, mientras que las pendientes leves $(0$ - $8^{\circ}$ ), no guardan relación con dichos eventos. Esto se explica porque mientras el ángulo de la pendiente aumenta también incrementa la tensión en el suelo o del material no consolidado favoreciendo deslizamientos (Cardozo, 2013). Adicionalmente las altas pendientes favorecen el arranque y movilización de material a las corrientes tributarias y de allí a la corriente principal.

El mapa de litología agrupa las diversas unidades litológicas que afloran en el área de la cuenca en veinte clases, siendo las lutitas silíceas y calcáreas, con intercalaciones de calizas y areniscas las que presentan una mayor correlación con la generación de flujos de detritos, seguidas de las matrices arcillosas con bloques heterométricos de lutitas y calizas; las Chert, liditas y limolitas silíceas y los suelos arcillosos con limos y arenas. La cobertura es la variable cuyas clases tienen los valores más altos de correlación, por encima de todas las demás clases de las otras variables consideradas, encontrando valores muy altos de correlación en la generación de flujos de detritos para los cultivos de yuca, frutales, maíz y los suelos desnudos. Esto puede deberse a que la eliminación de la vegetación nativa para establecer cultivos tiende a generar inestabilidad en el estrato superficial, dado que parte de la estabilidad en pendientes fuertes se debe al enraizamiento (Flórez, 1986). El mapa de curvatura indica que la mayor población de flujos de detritos está relacionada con la curvatura cóncava o convexa y no con sectores planos. En tal sentido, hay una asociación ligeramente mayor de la curvatura cóncava a la generación de flujos, que podría deberse a que cuando la curvatura de un determinado sector de ladera es cóncava, el flujo de agua se concentra en dicha hondonada, lo que aumentará tanto el contenido de humedad del suelo como el tiempo que permanecerá saturado, pudiendo así generar erosión y desestabilización del estrato superficial.

En la zona de estudio el espesor del estrato superficial varía entre $0.28 \mathrm{~m}$ y $14 \mathrm{~m}$, encontrando que las profundidades entre $2.81 \mathrm{~m}$ y $10.18 \mathrm{~m}$ son las que guardan mayor asociación con la ocurrencia de flujo de detritos, mientras que para espesores menores a $2.81 \mathrm{~m}$ no se encuentra correlación. De acuerdo con Remondo (2001) este comportamiento puede deberse a que los mayores espesores de formación superficial, pueden reducir el efecto estabilizador de las raíces y favorecer la inestabilidad, siendo determinantes en la capacidad de almacenamiento de agua en el terreno, favoreciendo mayores presiones de poros y aumentando el peso de la masa.

El modelo FLO-2D simula el movimiento de flujo y el comportamiento de la depositación de los flujos de detritos, logrando precisar las zonas de amenaza, en función de la intensidad del evento y la probabilidad de ocurrencia. El $35.2 \%$ del casco urbano de Útica se encuentra en zona de amenaza media. La amenaza media se encuentra focalizada principalmente sobre las rondas de la Q. la Negra y del Río Negro. El 64.8\% del casco urbano se encuentra en zona de amenaza baja. El índice de resistencia de las edificaciones presenta similitudes ya que la gran mayoría de las edificaciones inventariadas están construidas en mampostería (ladrillo y bloque), son de un solo piso y presentan similitudes en la disposición y características de las aberturas de fachada 
Elíndice de exposición presenta amplias variaciones, siendo más alto en las edificaciones que se encuentran cercanas a la Q. la Negra y al Río Negro y menores valores hacia el centro de la población, esto obedece a que las mayores alturas de flujo y presiones de impacto se encuentran sobre las edificaciones que están en la ronda del cauce de los dos cuerpos de agua. El riesgo de los elementos expuestos, al materializarse la amenaza se presenta como una función de la amenaza, vulnerabilidad y el costo asociado al elemento expuesto. Este tipo de evaluación sirve como herramienta de planeación para realizar análisis de costo-beneficio e implementación de medidas de reducción del riesgo.

\section{Conclusiones}

Al hacer el análisis de umbrales de lluvia detonante de flujo de detritos, bien sea con metodologías probabilísticas o determinísticas, estas deben ser integradas a la susceptibilidad del terreno, para establecer adecuadamente el modelo de pronóstico de flujos de detritos con base en condiciones geomorfológicas (litología, pendiente, espesor estrato superficial), de uso del suelo y morfodinámica (inventario de procesos), entre otras.

La metodología presentada unifica criterios de evaluación tanto para amenaza como para vulnerabilidad y riesgo por flujos de detritos detonados por lluvia, que de acuerdo con la revisión bibliográfica, hasta la fecha no se habían integrado en un documento y solo se habían evaluado aisladamente. Esta metodología permite desarrollar protocolos de actuación en caso de desastre y resulta ser una herramienta útil para los especialistas al momento de diseñar medidas de contención y/o mitigación que contribuyan a reducir pérdidas y escoger posibles localizaciones para la construcción de nuevas infraestructuras.

\section{Referencias}

Acosta, E. (2006). Estudio geomorfológico y estimación de la susceptibilidad a flujos de derrubios y desprendimientos de rocas en el Valle de Benasque. Tesis de doctorado, Instituto Geológico y Minero de España, Zaragoza

Blais-Stevens, A. and Behnia, P. (2016). Debris flow susceptibility mapping using a qualitative heuristic method and Flow-R along the Yukon Alaska Highway Corridor, Canada. Natural Hazards and Earth System Sciences 16, 449-462
Bonham-Carter, G.F. (1994). Geographic information systems for geoscientists, modeling with GIS. Pergamon Press, Oxford

CAR (2013). Diagnóstico de la cuenca Quebrada La Negra. Bogota D.C. Corporación Autónoma Regional de Cundinamarca Cardozo, C.P. (2013). Zonación de susceptibilidad por procesos de remoción en masa en la cuenca del río Tartagal, Salta (Argentina). Tesis de Magíster, Universidad Nacional de Córdoba

Castellanos, R. y González, A. (1996). Relaciones entre la lluvia anual y la lluvia crítica que dispara movimientos en masa. IX Jornadas Geotécnicas de la Ingeniería Colombiana, Santa Fe de Bogotá, $4.62-4.70$

Comunidad Andina (2009). Atlas de las dinámicas del territorio andino: población y bienes expuestos a amenazas naturales. Cali, Corporación OSSO

Chaparro, O.I. (2005). Evaluación de riesgo por flujo de lodo en la Quebrada La Chapa, Municipios de Tasco y Socha-Boyacá. Tesis de doctorado, Universidad Nacional de Colombia

Esper, M.Y. (2014). Debris flow susceptibility mapping in a portion of the Andes and Preandes of San Juan, Argentina using frequency ratio and logistic regression models. Earth Sciences Research Journal 17, No. 2, 159-167

Esper, M.Y. (2012). Análisis de la vulnerabilidad por flujos en masa en la provincia de San Juan (oeste de Argentina). Revista de la Sociedad Geológica de España 25(3-4), 145-156

Esper, M.Y. (2011). Inventario de procesos de remoción en masa en un sector del Departamento Iglesia, San Juan, Argentina. Revista de la Asociación Geológica Argentina 68, 225-232

Fídel, L. y Zavala, B. (2013). Susceptibilidad a los movimientos en masa en la cuenca de la quebrada Hualanga. XIII Congreso Peruano de Geología, 97-122

Flórez, A. (1986). Geomorfología del área Manizales-Chinchiná, Cordillera Central, Colombia. Análisis Geográficos 9, 1-58

García, R. and López, J.L. (2005). Debris flows of December 1999 in Venezuela. In Debris-flow Hazards and Related Phenomena, Springer, 519-538

García, R., López, J.L., Noya, M., Bello, M., González, N. Paredes, G. and Vivas, M. (2002). Mapas de riesgo para eventos de flujo de barro y detritos en el estado de Vargas y Caracas. Informe proyecto Ávila, Caracas, Venezuela 
Gómez, N., Osorio, Y. y Salazar, J. (2013). SIG para determinar la susceptibilidad a movimientos en masa en a cuenca del río Campoalegre. Trabajo de grado, Universidad de Manizales

González, A. y Millán, J. (1998). Procedimiento para la evaluación de la amenaza por fenómenos de remoción en masa en Santafe de Bogotá-Colombia. INGEOCIM

Haigh, M.J., Rawat, J.S. and Bartarya, S. (1988). Environmental correlations of landslides frequency along new highways in the Himalaya: preliminary results. Catena 15(6), 539-553

ISSMFE (1997). Manual for zonation on areas susceptible to rain induced slope failure. Asian Technical Committee on Geotechnology for Natural Hazards, Japanese Geotechnical Society

Kanji, M.A., Massad, F. and Cruz, P.T. (2003). Debris flows in areas of residual soils: occurrence and characteristics. International Workshop on Occurrence and Mechanism of Flows in Natural Slopes and Earthfills, 1-13

Kanji, M.A., Cruz, P.T., Massad, F. and Araújo Filho, H.A. (2001). Environmental effects of debris flows and their protection measures. XV International Conference on Soil Mechanics and Geotechnical Engineering, Istanbul, Vol. 3: 1913-1916

Kanji, M.A., Cruz, P.T., Massad, F. and Araujo Filho, H.D. (1997). Basic and common characteristics of debris flows. 2nd Panamerican Symposium on Landslides, R. Janeiro, Vol.2: 223231

Keiler, M., Sailer, R., Jörg, P., Weber, C., Fuchs, S., Zischg, A. and Sauermoser, S. (2006). Avalanche risk assessment? a multi-temporal approach, results from Galtür, Austria. Natural Hazards and Earth System Science 6(4), 637-651

Lara, G. (2002). Análisis de amenaza por inestabilidad de la parte sur del municipio de Guacamayas. Departamento de Boyacá. Trabajo de grado en Geotecnia, Universidad Nacional de Colombia

Léone, F. (1996). Concept de vulnerabilité appliqué a l'évaluation des risques générés par les phénomènes de mouvements de terrain. Tesis de doctorado, Université de Grenoble (en francés)

Mardones, M. y Vidal, C. (2001). La zonificación y evaluación de los riesgos naturales de tipo geomorfológico: un instrumento para la planificación urbana en la ciudad de Concepción. Revista Latinoamericana de Estudios Urbano Regionales EURE 27(81), 97-122
Mayorga, R. (2003). Determinación de umbrales de lluvia detonante de deslizamientos en Colombia. Tesis de Magíster, Universidad Nacional de Colombia

Moreiras, S.M. (2004). Zonificación de peligrosidad y de riesgo de procesos de remoción en masa en el valle del río Mendoza. Provincia de Mendoza. Tesis doctoral, Universidad Nacional San Juan: 274 p.

Moreiras, S.M. (2009). Análisis estadístico probabilístico de las variables que condicionan la inestabilidad de las laderas en los valles de los ríos Las Cuevas y Mendoza. Revista de la Asociación Geológica Argentina 65(4), 780-790

Montero, J. (1997). Amenazas de origen natural en la región Andina y Colombia. III Simposio Panaméricano de deslizamientos, 419-459

Náquira Bazán, M.V. (2009). Susceptibilidad de remociones en masa en las costas de fiordos cercanos a Hornopirén, X Región. Memoria de título de Geólogo, Universidad de Chile

O'Brien, J.S. (2006). FLO-2D user's manual, version 2006.01. FLO-2D Software. Inc., Nutrioso

O’Brien, J., Julien, P. and Fullerton, T. (1993). Two-dimensional water flood and mudflow simulation. Journal of Hydraulic Engineering 119(2), 244-261

Papathoma-Koehle, M., Keiler, M., Totschnig, R. and Glade, T. (2012). Improvement of vulnerability curves using data from extreme events: debris flow event in South Tyrol. Natural Hazards 64(3), 2083-2105

Quan Luna, B., Blahut, J., van Westen, C.J., Sterlacchini, S., van Asch, T.W. and Akbas, S.O. (2011). The application of numerical debris flow modelling for the generation of physical vulnerability curves. Natural Hazards and Earth System Sciences 11(7), 20472060

Remondo , J. (2001). Elaboración y validación de mapas de susceptibilidad de deslizamientos mediante técnicas de análisis espacial. Tesis doctoral, Universidad de Oviedo

SNGRD (2014). Reporte del Sistema Nacional para la Gestión del Riesgo de Desastres en Colombia. Dirección de Gestión del Riesgo del Ministerio del Interior y de Justicia

Valderrama, P. (2006). Geología, geodinámica y simulación de flujos en las ciudades de Urubamba y Ollantaytambo. Tesis de pregrado, Universidad Nacional de San Antonio, Cusco, Perú 